\title{
A-Type Stars as a Unique Challenge in Time-Domain Studies
}

\author{
A.E. Lynas-Gray ${ }^{1}$ and G. Mathys ${ }^{2}$ \\ ${ }^{1}$ Department of Physics and Astronomy, University College London, \\ Gower Street, London WC1E 6BT, England \\ email: aelg@astro.ox.ac.uk \\ ${ }^{2}$ Joint ALMA Observatory \& European Southern Observatory, \\ 3107 Alonso de Cordova, Santiago, Chile \\ email: gmathys@eso.org
}

\begin{abstract}
As photometric standards, A-Type stars have proved to be extremely useful; this is particularly true of Vega, the fundamental photometric standard. The identification of at least some Main-Sequence A-type stars as small amplitude variables, while of interest as an issue of fundamental astrophysics, needs to be understood if they are to continue to be used as photometric standards. Flaring and Rossby waves are proposed in the literature as possible explanations as was discussed during a well attended debate, at the International Astronomical Union Symposium 339; this paper summarises the discussion and suggests future investigations.
\end{abstract}

Keywords. stars: early-type, stars: oscillations, stars: activity

\section{Introduction}

A-type Main-Sequence (MS) stars are unique, showing periodic variations on timescales ranging from minutes to centuries. As discussed below, variations are caused by different physical processes, including pulsation, rotation and multiplicity; they manifest themselves through observables, such as photometric brightness, spectral line strength and shape, radial velocity, as well as magnetic field strength and orientation. The diversity in timescales over which variability needs to be followed and characterised, along with diagnostics and techniques to be used to that effect, makes A-type stars particularly well suited to illustrate a range of challenges in time-domain astronomy. Most daunting among those challenges is to identify A-type stars constant enough to be used as photometric standards in the era of space-based photometry.

Space-based missions such as Kepler (Borucki et al. 2010) and K2 (Howell et al. 2014) have provided and continue to provide photometric data of unprecedented precision when compared with ground-based photometry. In particular, space-based photometry obtained over many months of nearly continuous observing provides light curves from which stars previously understood to be constant, are identified as very small amplitude variables. The quality of Kepler data allows Uytterhoeven et al. (2011) to present the first general characterisation of the pulsational behaviour of MS A-F type stars. While pulsation in MS A-type stars is of interest in so far as it admits asteroseismic studies, it is also crucial that it be properly understood in view of the important role MS A-type stars have as photometric standards.

A lively debate on the variability of MS A-type stars, and A-type stars in general, was held during the International Astronomical Union Symposium (IAUS) 339 on "Southern Horizons in Time-Domain Astronomy". In the present paper, we have tried to summarise the IAUS 339 discussion on A-type star variability. First we highlight the importance of 
the debate by briefly reviewing the role of MS A-type stars as photometric standards. Then we discuss A-type star variability due to starspots, flares and Rossby waves.

\section{Photometric Standards}

The success of Johnson \& Morgan's (1953) UBV photometric system and the bright A0 V star Vega having $V=0.03,(B-V)=0.00$ and $(U-B)=-0.01$ in this system, confirmed its well established status as the fundamental photometric standard. Several determinations of the Vega absolute spectral energy distribution were attempted by comparing monochromatic flux measurements with laboratory sources at near ultraviolet, optical and infrared wavelengths. Oke \& Schild (1970) provide an absolute spectral energy distribution for Vega from 3300 to $10800 \AA$ as earlier determinations exhibit discrepancies of as much as $10 \%$. Hayes \& Latham (1975) improve atmospheric extinction corrections and derive a better absolute spectral energy distribution for Vega. Of course it is preferable to have an absolute spectral energy distribution which does not depend on corrections for atmospheric extinction and for this reason Bohlin \& Gilliland (2004) use the Space Telescope Imaging Spectrograph to provide absolute spectrophotometry of Vega from 0.17 to $1.10 \mu$.

A shell around Vega which manifests itself as an infrared excess (Selby et al. 1983, Blackwell et al. 1983, Harvey et al. 1984, Aumann et al. 1984) casts doubt on its suitability as a fundamental photometric standard at wavelengths longer than $\sim 1 \mu$. At longer wavelengths, Cohen et al. (1992) establish a network of standard stars based on Astar models of Vega and Sirius, an approach which prompts Bohlin (2014) to provide space-based absolute spectrophotometry of Sirius as well as Vega. Bohlin et al. (2014) give a short review of absolute flux calibration from the ultraviolet to the mid-infrared, describing techniques and recent developments.

\section{Variability}

The use of A-type stars as photometric standards, and Vega as the fundamental photometric standard in particular, follows from the understanding that these are not active stars or indeed intrinsic variables. Any variability detected among the normal A-type stars would be of immediate interest because of their contribution to the establishment of standard photometric systems and absolute flux calibration of standard photometric systems. Koen's (2001) tentative identification of pulsation in early A-type stars, based on Hipparcos epoch photometry, resulted in further space-based photometry being anticipated with considerable interest.

Balona (2011) examines Quarter 0 and Quarter 1 Kepler light curves of more than 9000 A-F stars mostly obtained in the long cadence mode with exposure times of 29.4 minutes. The majority of A-F stars are found to vary with frequencies of $<5 \mathrm{~d}^{-1}$ and low amplitudes of 40-150 ppm. As there was at that time no known mechanism which could lead to low frequency pulsations in A stars, Balona (2011) investigates rotational modulation as a possible explanation and concludes that the light variations in A-type stars may be due to starspots or other co-rotating structures.

Using the full four-year Kepler dataset, Balona (2017) finds a high correlation between projected rotational velocities and photometric frequencies in a sample of thirty mid-A to late-B stars. Moreover, time-frequency diagrams show stochastic variations in all respects similar to those of spotted cool stars. Balona (2017) therefore proposes starspots, or some other co-rotating feature, to be the rule rather than the exception among A-type stars and the origin of light variability. Starspots could imply the presence of substantial localised 
magnetic fields, as discussed in more detail below, but chemical surface inhomogeneities or "chemical weather" may be an alternative explanation.

Kochukhov et al. (2005) and Hubrig et al. (2006), for example, detect variations in mercury lines (and those of some other elements) in the spectra of HgMn stars where magnetic fields are weak (Makaganiuk et al. 2012) or tangled (Kochukhov et al. 2013). Kochukhov et al. (2007) attribute such variations to chemical surface inhomogeneities; these processes, if active in other A-type stars, would account for a correlation between photometric frequencies and projected rotation speed. If "chemical weather" in the atmospheres of A-type stars bears some resemblance to terrestial weather as Kochukhov et al. (2007) argue, it would also be responsible for stochastic photometric variability.

Concern that Vega was being used as a fundamental photometric standard star and might itself be a variable goes back at least as far as Fath (1935) and contemporary studies he cites. Observations suggesting Vega is a small amplitude variable are also discussed by Fernie (1981) and Engelke et al. (2010). Bohlin et al. (2014) do not agree, however, with Engelke et al.'s conclusion that Hipparcos photometry shows Vega to be a variable, as saturation of the pulse counting electronics in the image dissector photomultiplier tube is regarded as a more probable explanation.

If Vega is a small amplitude variable, a possible explanation could be the co-rotating structures (starspots) which Böhm et al. (2015) discover. In a star with normal abundances like Vega, the origin of rotational modulation of surface structures must be different from the chemically peculiar Ap/Bp/HgMn stars. With a weak magnetic field and a high rotation rate, the atmosphere would not be stable enough for chemical anomalies to develop through atomic diffusion. If weak magnetic fields observed in Vega are responsible for starspots, these should be brighter than the photospheric average because dark spots only occur when the magnetic field is strong enough to limit convective heat transport within them.

Petit et al. (2017) use Doppler imaging to reconstruct a time-sequence of three maps of the visible Vega photosphere. While a number of surface features are seen in all three maps, others seem to evolve over the time-span of the observations, suggesting changes can affect the Vega surface on a time-scale of days or less. Although it is tempting to attribute starspots to the weak Vega magnetic field, which Vidal et al. (2018) suggest could have a tidal origin, further investigation is required.

\section{Stellar Flares}

Stellar flares are dramatic increases in brightness over almost the whole electromagnetic spectrum. A rapid increase in brightness is followed by a slow decline, the time-scale typically ranging from a few minutes to several hours. While stellar flares are usually associated with M-dwarfs (UV Ceti variables), they are also well-studied in the Sun. Energy released by the reconnection of magnetic field lines in the outer solar atmosphere, accelerates energetic particles into deeper atmospheric layers, resulting in a solar flare seen as emission from X-rays to radio waves (Benz \& Güdel 2010). Although flares in active M-dwarfs may be as much as $\sim 10^{3}$ more energetic than solar flares (Güdel \& Nazé 2009), they are understood to form in essentially the same way as in the Sun.

Although what constitutes a "flare" is perhaps not as well-defined as it should be, Balona (2012) is guided by the above-mentioned description in his examination of Kepler light curves for nearly 2000 A-type stars and detects flares in nineteen of them. The energy released in an A-type star flare is estimated by Balona to be $\sim 10^{36}$ ergs which is difficult to understand as A-type stars have weak magnetic fields. A comparison with energies released in cool star flares, also observed by Kepler, leads Balona (2012) to conclude that 
flares attributed to the nineteen A-type stars do indeed originate in those stars and not in an apparent or physical cool companion.

Balona (2013) identifies additional A-type stars in the Kepler field which show flares and concludes that A-type stars are active and, like cooler stars, have starspots and flares. On considering flare stars across the Hertzsprung-Russell Diagram, Balona (2015) finds the proportion of MS stars which flare to be essentially independent of effective temperature but energies released during a flare are strongly correlated with stellar luminosity and radius. Furthermore, flare stars are found to have larger spots and higher rotation rates than non-flare stars. While rotation plays a role in generating flares, Balona (2015) notes that this may be indirect; any possibility that it and differential rotation have a direct role, needs further investigation.

Pedersen et al. (2017) critically reexamine Balona's (2012) claim to have identified flares in Kepler light curves of A-type stars, arguing that for stars to flare they have to have a sufficiently deep outer convection zone, strong large-scale magnetic fields or strong radiatively driven winds; normal A-type stars are understood to have none of these and therefore should not flare. In cases where Pedersen et al. obtain spectra of Balona's (2013) targets, these are confirmed to be A-type stars and some are spectroscopic binaries. Alternative explanations for observed flares are found in at least nineteen of the thirtythree A-type stars for which Balona finds flaring and Pedersen et al. do not identify a convincing target supporting the conclusion that A-type stars flare. A weakness in the Pedersen et al. analysis is that they decline to address Balona's (2015) finding of a strong correlation between energies released during a flare and stellar luminosity and radius.

Van Doorsselaere et al. (2017) develop an automated flare and characterisation algorithm which they apply to Kepler (Quarter 15) raw light curves. A further twenty-four A-type stars which flare are detected and those which Pedersen et al. (2017) disqualify as flaring A-type stars are also recovered by the Van Doorsselaere et al. study, as would be expected. A consequence of the Van Doorsselaere et al. study is that it would be premature to dismiss the possibility of flaring in A-type stars.

\section{Rossby Waves}

Platzman (1968) reviews early work on Rossby waves in the context of meteorology. In rotating stars, global normal modes of Rossby waves (Papaloizou \& Pringle 1978, r-modes) couple with the spheroidal motion caused by the Coriolis force to produce temperature perturbations and consequently stellar variability. Saio et al. (2018) provide evidence, and supporting theory, for global Rossby waves in upper main-sequence stars. In amplitude spectra of spotted early A to B-type stars, Saio et al. find groups of symmetric (with respect to stellar equators) r-mode frequencies just below the frequency of a structured peak which they suggest represents an approximate stellar rotation rate.

Similar amplitude-spectra for spotted A-type stars are obtained from Kepler data by Balona (2017, his figure 7). Balona considers the broad spread of peaks, which Saio et al. identify as r-mode frequencies, to be a consequence of strong differential rotation and abundant solar-like spots. The structured sharp peak which Saio et al. consider to represent an approximate stellar rotation rate is characterised by Balona (2017) as fully resolved, and due to reflection from an orbiting Jupiter-sized planet.

Rossby waves by their nature must be expected in the amplitude-spectra of rotating stars, and their amplitudes will clearly be dependent on rotation speed. The Saio et al. (2018) interpretation of theoretical amplitude-spectra of rotating A-type stars on the MS corresponds, in a natural way, with a variability that would appear to be co-rotating with the star. A possible confusion with starspots or other co-rotating features in the stellar 
photosphere could then occur in an obvious way. Current stellar models (Kallinger \& Matthews 2010) suggest that A-type stars on the MS have a thin convection zone and do not therefore form starspots.

\section{Future Directions}

In many cases stellar models, and their convection zones in particular, are reliant on Böhm-Vitense's (1958) Mixing-Length theory (MLT) which has a free parameter (the mixing-length) for which a calibration is required. One of many attempts to replace MLT is by Pasetto et al. (2014) who present a self-consistent, scale-free convection (SFC), analytical formulation starting from a conventional solution of the Navier-Stokes/Euler equation (in effect the Bernoulli equation for a perfect fluid) expressed in a non-inertial reference frame co-moving with convective elements. MLT and SFC comparisons (Pasetto et al. 2016) are encouraging, with good agreement being found for MS models. But as Arnett et al. 2015) point out, stars are much more complex than the simplified onedimensional models. When computer-power becomes adequate, and well-resolved threedimensional simulations of whole stars for evolutionary time-scales have been computed, a better understanding of convection in A-type MS stars should become available. Until then, it would be premature to assert that A-type MS stars do not flare or have starspots because their convection zones are not sufficiently extensive.

As mentioned above, the study by Van Doorsselaere et al. (2017) suggests further work is needed to establish whether A-type MS stars flare. The thirty-three A-type flare stars which Balona $(2012,2013)$ identifies and the additional twenty-four which Van Doorsselaere et al.(2017) find should in due course be observed by the Large Synoptic Survey Telescope Tyson (2002, LSST). The higher resolution which the LSST cameras achieve would identify $\mathrm{M}$ dwarf flare stars observed in the same Kepler pixels as A-type stars which Balona and Van Doorsselaere et al. declare to be flare stars; the origin of the flare could then be established with more confidence, although the LSST would not necessarily resolve all objects contaminating a $3.98^{\prime \prime} \times 3.98^{\prime \prime}$ Kepler pixel.

Rossby waves also produce light variations in stars which are rotating rapidly; these result from temperature and pressure variations in the photosphere and a corresponding radial velocity perturbation should also be expected. It would therefore be of some interest to estimate the Rossby wave contribution to Vega line profile variations which Böhm et al. (2015) observe. The Böhm et al. discovery of starspots on Vega would seem to have been secured through Doppler imaging by Petit et al. (2017). But it would for example be worthwhile to investigate whether any underlying physics distinguishes transient and consistently recovered surface features in Petit et al. maps. Could the transient features be due to spots, and those consistently seen in all three maps be a manifestation of Rossby waves?

While the Bohlin (2014) flux calibration based on Sirius and Vega remains the best available flux standard, its limitations need to be explored by studying Sirius in the way Böhm et al. (2015) and Petit et al. (2017) study Vega. The importance of doing so is emphasised by the Petit et al. (2011) detection of a weak surface magnetic field on Sirius A. Almost as important is the need to extend the Böhm et al. and Petit et al. study to the eighteen new A-type flux standards which Allende Prieto \& del Burgo (2016) propose. 


\section{References}

Allende Prieto, C., \& del Burgo, C. 2016, MNRAS, 455, 3864

Arnett, W. D., Meakin, C., Viallet, M., Campbell, S. W., Lattanzio, J. C., \& Mocák, M. 2015, ApJ, 809, 30

Aumann, H. H., Beichman, C. A., Gillett, F. C., et al. 1984, ApJ (Letters), 278, L23

Balona, L. A. 2011, MNRAS, 415, 1691

- 2012, MNRAS, 423, 3420

- 2013, MNRAS, 431, 2240

- 2015, MNRAS, 447, 2714

- 2017, MNRAS, 467, 1830

Benz, A. O., \& Güdel, M. 2010, ARAA, 48, 241

Blackwell, D. E., Leggett, S. K., Petford, A. D., Mountain, C. M., \& Selby, M. J. 1983, MNRAS, 205,897

Bohlin, R. C. 2014, AJ, 147, 127

Bohlin, R. C., \& Gilliland, R. L. 2004, AJ, 127, 3508

Bohlin, R. C., Gordon, K. D., \& Tremblay, P.-E. 2014, PASP, 126, 711

Böhm, T., Holschneider, M., Lignières, F., et al. 2015, A\&A, 577, A64

Böhm-Vitense, E. 1958, ZfA, 46, 108

Borucki, W. J., Koch, D., Basri, G., et al. 2010, Science, 327, 977

Cohen, M., Walker, R. G., Barlow, M. J., \& Deacon, J. R. 1992, AJ, 104, 1650

Engelke, C. W., Price, S. D., \& Kraemer, K. E. 2010, AJ, 140, 1919

Fath, E. A. 1935, Lick Observatory Bulletin, 17, 115

Fernie, J. D. 1981, PASP, 93, 333

Güdel, M., \& Nazé, Y. 2009, A\& AR, 17, 309

Harvey, P. M., Wilking, B. A., \& Joy, M. 1984, Nature, 307, 441

Hayes, D. S., \& Latham, D. W. 1975, ApJ, 197, 593

Howell, S. B., Sobeck, C., Haas, M., et al. 2014, PASP, 126, 398

Hubrig, S., González, J. F., Savanov, I., Schöller, M., Ageorges, N., Cowley, C. R., \& Wolff, B. 2006, MNRAS, 371, 1953

Johnson, H. L., \& Morgan, W. W. 1953, ApJ, 117, 313

Kallinger, T., \& Matthews, J. M. 2010, ApJ (Letters), 711, L35

Kochukhov, O., Adelman, S. J., Gulliver, A. F., \& Piskunov, N. 2007, Nature Physics, 3, 526

Kochukhov, O., Makaganiuk, V., Piskunov, N., et al. 2013, A\&A A, 554, A61

Kochukhov, O., Piskunov, N., Sachkov, M., \& Kudryavtsev, D. 2005, A\&\&A, 439, 1093

Koen, C. 2001, MNRAS, 321, 44

Makaganiuk, V., Kochukhov, O., Piskunov, N., et al. 2012, A\&AA, 539, A142

Oke, J. B., \& Schild, R. E. 1970, ApJ, 161, 1015

Papaloizou, J., \& Pringle, J. E. 1978, MNRAS, 182, 423

Pasetto, S., Chiosi, C., Chiosi, E., Cropper, M., \& Weiss, A. 2016, MNRAS, 459, 3182

Pasetto, S., Chiosi, C., Cropper, M., \& Grebel, E. K. 2014, MNRAS, 445, 3592

Pedersen, M. G., Antoci, V., Korhonen, H., White, T. R., Jessen-Hansen, J., Lehtinen, J., Nikbakhsh, S., \& Viuho, J. 2017, MNRAS, 466, 3060

Petit, P., Hébrard, E. M., Böhm, T., Folsom, C. P., \& Lignières, F. 2017, MNRAS, 472, L30

Petit, P., Lignières, F., Aurière, M., et al. 2011, A\& $A$, 532, L13

Platzman, G. W. 1968, Quarterly Journal of the Royal Meteorological Society, 94, 225

Saio, H., Kurtz, D. W., Murphy, S. J., Antoci, V. L., \& Lee, U. 2018, MNRAS, 474, 2774

Selby, M. J., Mountain, C. M., Blackwell, D. E., Petford, A. D., \& Leggett, S. K. 1983, MNRAS, 203, 795

Tyson, J. A. 2002, in: J. A. Tyson, \& S. Wolff (eds.), Survey and Other Telescope Technologies and Discoveries, Proceedings of SPIE, vol. 4836, 10.

Uytterhoeven, K., Moya, A., Grigahcène, A., et al. 2011, AESA, 534, A125

Van Doorsselaere, T., Shariati, H., \& Debosscher, J. 2017, ApJS, 232, 26

Vidal, J., Cébron, D., Schaeffer, N., \& Hollerbach, R. 2018, MNRAS 